\title{
Estudio y diseño del Sistema de Tierra Integral de la Facultad de Ingeniería de la Universidad Autónoma de Campeche
}

\section{Study and design of the Integral Grounding System of the Faculty of Engineering of the Campeche's Autonomous University}

\author{
LEZAMA-ZÁRRAGA, Francisco Román†๋*, OVANDO-SIERRA, Juan Carlos, CASTILLO-TÉLLEZ, \\ Margarita y ANDRADE-DURÁN, Juan Edgar
}

Universidad Autónoma de Campeche, Campus V, Predio s/n por Av. Humberto Lanz Cardenas y Unidad Habitacional Ecologica Ambiental, Col. Ex-Hacienda Kala, San Francisco de Campeche, Cam., México. CP 24085

ID $1^{\text {er }}$ Autor: Francisco Román, Lezama-Zárraga / ORC ID: 0000-0003-3397-7881, Researcher ID Thomson: U-12292018, CVU CONACYT ID: 205493

ID $1^{\text {er }}$ Coautor: Juan Carlos, Ovando-Sierra / ORC ID: 0000-0003-4358-6657, Researcher ID Thomson: S-2357-2018, CVU CONACYT ID: 358434

ID $2^{\text {do }}$ Coautor: Margarita, Castillo-Téllez / ORC ID: 0000-0001-9639-1736, Researcher ID Thomson: S-2283-2018, CVU CONACYT ID: 210428

ID $3^{\text {er }}$ Coautor: Juan Edgar, Andrade-Durán / ORC ID: 0000-0002-7370-1290, Researcher ID Thomson: T-8830-2018, CVU CONACYT ID: 238526

DOI: $10.35429 /$ JEE.2019.10.3.8.16

Recibido 20 Junio, 2019; Aceptado Septiembre 30, 2019

\section{Resumen}

Hoy en día, los sistemas eléctricos en baja tensión son de mayor complejidad debido a cargas no lineales sensibles a las variaciones de tensión por perturbaciones o mala calidad de la energía en la red eléctrica de suministro, por lo que es necesario proteger los equipos y a las personas que a diario utilizan estas instalaciones eléctricas. En este artículo se presenta un estudio para diseñar el sistema de tierra equipotencial de la Facultad de Ingeniería de la Universidad Autónoma de Campeche con el fin de obtener un valor de impedancia de tierra que cumpla con la normas NXM-J-549-ANCE-2005 y NOM-001-SEDE-2012 para proporcionar condiciones de seguridad a las personas y equipos que operan en la instalación eléctrica en baja tensión, drenando rápidamente la falla, logrando que el valor del voltaje con respecto a la tierra que se alcance en la parte fallada sea igual o inferior al valor de seguridad y ofrecer las medidas correctivas para mejorar la calidad de la energía, que recibimos de la empresa suministradora. Este estudio es la base para implementar un modelo de sistema de tierra integral para cualquier tipo de edificios de nuestro entorno para lograr niveles de voltaje estable a frecuencia constante.

Baja impedancia de tierra, Equipotencial, Voltaje estable

\begin{abstract}
Nowadays, low voltage electrical systems are more complex due to sensitive non-linear loads to voltage variations due to disturbances or poor quality of power in the electricity supply network, so it is necessary to protect equipment people who use these electrical installations every day. This paper presents a study to design the equipotential grounding system of the Faculty of Engineering of the Campeche's Autonomous University in order to obtain an earth impedance value that complies with the standards NXM-J-549-ANCE-2005 and NOM001-SEDE-2012 to provide safety conditions to people and equipment operating in the electrical installation in low voltage, quickly draining the fault, making the value of the voltage with respect to the earth reached in the part failure is equal to or less than the safety value and offer the corrective measures to improve the quality of the energy, which we receive from the supplier company. This study is the basis to implement a model of integral earth system for any type of buildings in our environment to achieve stable voltage levels at a constant frequency.
\end{abstract}

Low earth impedance, Equipotential, Stable voltage

Citación: LEZAMA-ZÁRRAGA, Francisco Román, OVANDO-SIERRA, Juan Carlos, CASTILLO-TÉLLEZ, Margarita y ANDRADE-DURÁN, Juan Edgar. Estudio y diseño del Sistema de Tierra Integral de la Facultad de Ingeniería de la Universidad Autónoma de Campeche. Revista de Ingeniería Eléctrica. 2019. 3-10: 8-16

\footnotetext{
$\dagger$ Investigador contribuyendo como primer Autor.

*Correspondencia al Autor correo electrónico: (frlezama@uacam.mx)
} 


\section{Introducción}

En la actualidad un sistema eléctrico en alta, media y baja tensión requiere ciertas características como la calidad, la confiabilidad, la continuidad y la seguridad para que opere de manera estable y brinde un servicio óptimo a los consumidores residenciales, comerciales, industriales y de servicios. Como parte de la seguridad de un sistema existe el sistema de puesta a tierra para asegurar la protección de las instalaciones y equipo eléctrico y sobre todo salvaguardar la integridad física de los operadores de dichas instalaciones y equipos cuando se presenten fallas en el sistema provocando cortocircuitos.

La puesta a tierra de equipos consiste en conectar a tierra las partes metálicas no conductoras de corriente que alojan a los sistemas o equipos y aparatos de utilización de energía eléctrica (Velasco, 2007). En la figura 1 se observa la interconexión a tierra de todos los medios de canalización metálicos, gabinetes metálicos, estructuras que soporten equipos eléctricos, carcazas de motores y generadores, tanques metálicos de transformadores y en general todas las cubiertas metálicas.

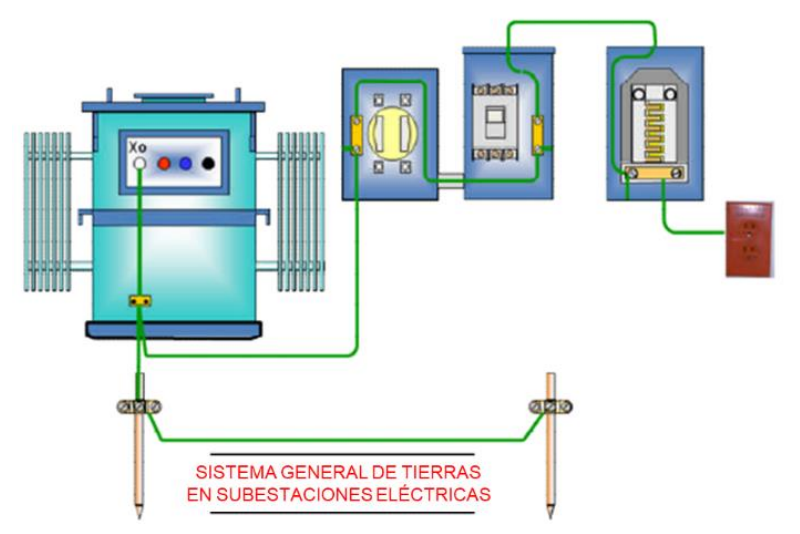

Figura 1 Puesta a tierra del sistema eléctrico

Fuente: Jesúss Velasco Solís, Entendiendo el sistema de tierras, México, 2007)

Para el diseño, se requieren cálculos muy precisos por lo que es necesario e indispensable interpretar adecuadamente las normatividades vigentes tales como la NOM-001-SEDE-2012, NXM-J-549-ANCE-2005 y la IEEE-Std-802000 ya que se trata de un sistema de seguridad para el ser humano. La Facultad de Ingeniería de la Universidad Autónoma de Campeche cuenta con un sistema eléctrico de distribución en media y baja tensión con un conductor de puesta a tierra.
Esta es la parte de la Norma Oficial Mexicana, en su Artículo 250, que específica cual es el conductor de nuestras instalaciones eléctricas que debe estar puesto a tierra. En este artículo, se verifica las condiciones iniciales del sistema de puesta a tierra, mediante la medición de resistividad del terreno y el valor de impedancia del conductor y los electrodos del sistema de puesta a tierra. Al existir una problemática por no tener los valores de resistencia de puesta a tierra que exigen las normas, se proponen acciones de mejoras al sistema de puesta a tierra para alcanzarlos. Y finalmente si se implementan las mejoras, se verifica de nuevo con mediciones si se alcanzaron los valores adecuados de baja impedancia del sistema de puesta a tierra.

En la primera sección del artículo, se explica de forma breve la importancia de tener un sistema de puesta a tierra confiable y seguro, los elementos y equipos eléctricos que deben estar conectados a tierra y el alcance de este trabajo. En la segunda sección, se describe la metodología propuesta para tener resultados significativos de eficiencia energética en la iluminación del campus. En la tercera sección, indica el procedimiento de las mediciones realizadas de acuerdo con la NOM vigente. Por último, en la cuarta sección se muestran los resultados obtenidos de mediciones e implementación de estrategias de eficiencia energética. Finalmente, en la sección de conclusiones se indica la importancia de este estudio y los resultados obtenidos

\section{Objetivos de la puesta a tierra}

El objetivo principal de un sistema de puesta a tierra es la protección de las personas, los equipos e instalaciones contra tensiones peligrosas y al mismo tiempo dar confiabilidad y continuidad al servicio eléctrico, creando para esto un circuito de retorno de muy baja impedancia para la circulación a tierra de corrientes de falla a tierra, corrientes producidas por electricidad estática, corrientes circulantes indeseables y las producidas por descargas atmosféricas. Otro objetivo fundamental de la puesta a tierra es limitar el potencial entre las partes no conductoras de corriente, que existan en la instalación, y entre esas partes y tierra para asegurar valores abajo de todas las condiciones de operación del sistema tanto normales como anormales. 
El peligro para el personal existe al mismo tiempo en que ocurra una falla a tierra. $\mathrm{Si}$ se fuerza a la corriente a fluir a través de una impedancia elevada la conexión a tierra puede crear diferencias de potencial peligrosas que puedan dañar al personal y equipo que se localicen cerca de dichos sistemas.

Todos los conceptos involucrados en los sistemas de tierra y la conexión de equipo son reglamentados y normalizados, por lo que se debe tomar en cuenta que este sistema no es simplemente uno más que hay que instalar en los edificios, comercios, industria y hasta en nuestros hogares para el funcionamiento de todo equipo eléctrico, sino también hay que pensar que se trata de un sistema de seguridad para el ser humano, es por esta razón que debemos darle la importancia que merece, para ello es necesario e indispensable conocer e interpretar adecuadamente la Norma Oficial Mexicana (NOM-001-SEDE-2012) actual, que en sus Artículos 250 y 921 tratan lo referente a la Puesta a Tierra.

El conductor puesto a tierra inicial en las cinco subestaciones es un conductor calibre 2 AWG para un sistema trifásico a cuatro hilos, 220/127 Volts conectado a una varilla copperweld de cobre de $1 / 2$ pulgada de diámetro y 1.5 metros de longitud. Este conductor puesto a tierra es instalado con la plena intención de conducir la corriente de desbalanceo del sistema (corriente en el neutro o retorno del sistema).

\section{Objetivo de la investigación}

Diseñar el Sistema de Tierra Integral equipotencial de la Facultad de Ingeniería de la Universidad Autónoma de Campeche aplicando la norma oficial mexicana NMX-j-549-ANCE2005 que cumpla las especificaciones de diseño, los métodos de cálculo, los materiales y tipos de mediciones con el fin de disminuir el riesgo de daño para las personas, equipos e instalaciones eléctricas en baja tensión cuando se presente una falla.

\section{Metodología propuesta}

Tomando como referencia la metodología de Checkland (Checkland, 1994), la propuesta consta de las siguientes etapas:
1. Preparación de herramientas, equipo de medición y del personal que realizará las mediciones en campo.

2. Levantamiento físico en las subestaciones con ayuda de los planos eléctricos, identificando cada uno de los conductores y electrodos de puesta a tierra y la trayectoria efectiva de puesta a tierra hacia los tableros de distribución y para los circuitos de alimentación y circuitos derivados.

3. Evaluar los niveles de resistividad del terreno e impedancia del conductor de puesta a tierra en cada subestación, de acuerdo con la NOM-001-SEDE-2012, en su artículo 250 y con la IEEE-Std-812012.

4. Analizar la información obtenida con ayuda de hojas de cálculo que incluyan las ecuaciones para el diseño de sistemas de puesta a tierra.

5. Definir las propuestas de mejora, a través de un informe, que proporcionarán los valores de baja impedancia del conductor de puesta a tierra para cada subestación e interconexión entre dichos conductores para mantener un sistema equipotencial (sistema integral de puesta a tierra).

6. Realizar una evaluación final de las condiciones finales del sistema, luego de haberse implementado las propuestas de mejora con el fin de verificar si el sistema de puesta a tierra interconectado $y$ equipotencial tiene condiciones óptimas de confiablidad y seguridad para los seres humanos y protección de equipos eléctricos.

La figura 2 ilustra las etapas de la metodología propuesta en este artículo.

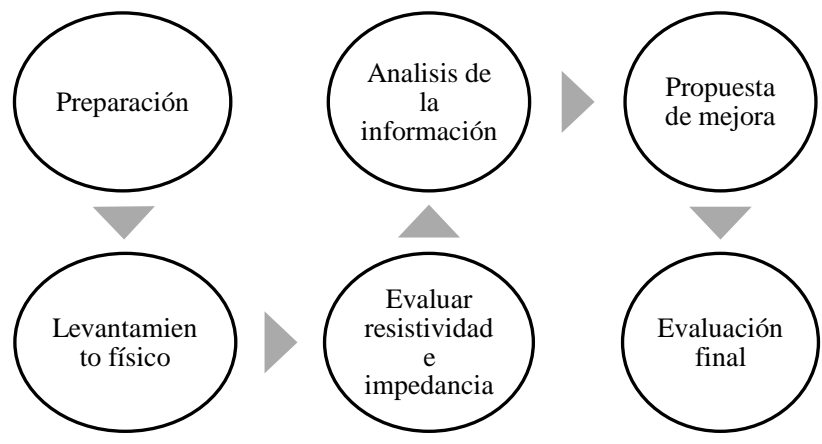

Figura 2 Etapas de la metodología de un sistema de puesta a tierra integral

Fuente: Elaboración Propia 


\section{El sistema de tierra física existente}

El sistema de tierras en cada una de las cinco subestaciones de la Facultad de Ingeniería consta de una varilla copperweld de $1 / 2$ pulgada de diámetro de cobre, las cuales están aisladas, sin interconexión entre ellas (ver figura 3).

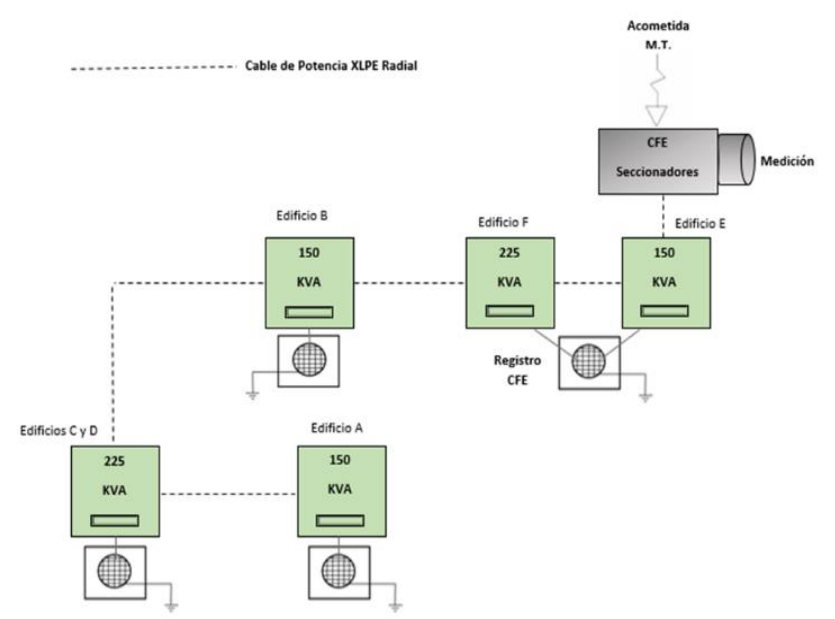

Figura 3 Sistemas de tierra en la Facultad de Ingeniería Fuente: Elaboración Propia

La tensión en las subestaciones es de 13.8 $\mathrm{kV}$ en el lado primario y $220 / 127 \mathrm{~V}$ en el lado secundario, conexión delta-estrella aterrizado.

\section{Medición de la resistividad del terreno}

El parámetro eléctrico del terreno que cambia de acuerdo al tipo de suelo y a la cantidad de agua que contiene es la resistividad $(\Omega-m$,). Algunos valores típicos se muestran en la siguiente tabla.

\begin{tabular}{|c|c|c|}
\hline \multirow{2}{*}{ Tipo de terreno } & \multicolumn{2}{|c|}{$\begin{array}{l}\text { Resistividad del terreno } \\
{[\Omega-\mathrm{m}]}\end{array}$} \\
\hline & $\begin{array}{l}\text { Margen de } \\
\text { valores }\end{array}$ & $\begin{array}{l}\text { Valor } \\
\text { medio }\end{array}$ \\
\hline Terreno pantanoso & $2-50$ & 30 \\
\hline Barro mezclado con paja & $2-200$ & 40 \\
\hline Terreno fangoso y arcilloso & $20-260$ & 100 \\
\hline Arena y terreno arenoso & $50-3000$ & $\begin{array}{r}200 \\
\text { (húmedo) } \\
\end{array}$ \\
\hline Turba & $>1200$ & 200 \\
\hline Grava (húmeda) & $50-3000$ & $\begin{array}{r}1000 \\
\text { (húmedo) } \\
\end{array}$ \\
\hline Terreno pedregoso y rocoso & $100-8000$ & 2000 \\
\hline $\begin{array}{l}\text { Hormigón: } 1 \text { parte de } \\
\text { cemento }+3 \text { partes de arena }\end{array}$ & $50-300$ & 150 \\
\hline $\begin{array}{l}1 \text { parte de cemento }+5 \\
\text { partes de grava }\end{array}$ & $100-8000$ & 400 \\
\hline
\end{tabular}

Tabla 1 Resistividad de varios suelos Fuente: Markiewicz, H. \& Klajn, A
Las dimensiones del lugar que alberga cada subestación son de 4.0 m. x 3.0 m., y está localizado sobre un terreno de tierra de arcilla y una capa inferior de caliza, con una resistividad promedio de $\rho=21.85 \Omega-\mathrm{m}$. de acuerdo a las mediciones realizadas.

Se utilizó un medidor de resistencias de tierra digital marca KOBAN modelo KRT-014P y se aplicó el método de tres puntos o Método de variación de profundidad (IEEE, 2000). La figura 4 ilustra las conexiones que deben realizarse al momento de la prueba.

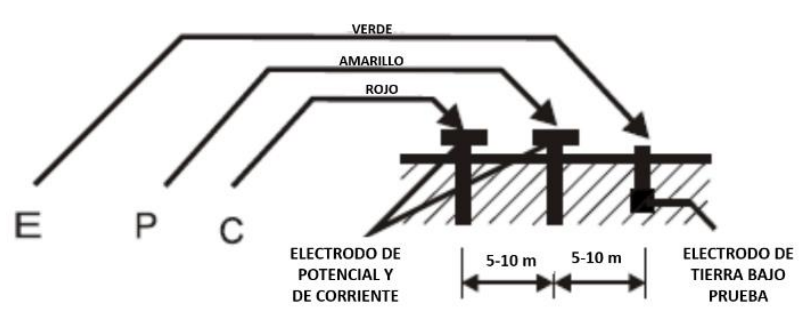

Figura 4 Conexiones en el método de los tres puntos Fuente: IEEE-Std-80-2000.

Este método consiste en medir la resistencia de puesta a tierra de un electrodo tipo varilla y calcular la resistividad del suelo basado en la ecuación de resistencia a tierra de un electrode

$\rho=\frac{2 \pi L R}{\ln \left(\frac{8 L}{D}\right)-1}$

donde:

$\mathrm{L}=$ distancia entre electrodos, en $\mathrm{m}$.

$\mathrm{R}=$ medición de resistencia, en Ohms

$\mathrm{D}=$ diámetro del electrodo tipo varilla, en $\mathrm{m}$.

E, P y C: Electrodo tipo varilla, electrodo auxiliar de potencial y electrodo auxiliar de corriente respectivamente.

En nuestro caso, el valor promedio de resistencia que midió el equipo es de $8.4 \Omega$, con una distancia entre electrodos de $3 \mathrm{~m}$. y con un diámetro de la varilla de $0.013 \mathrm{~m}$. así, obtuvimos el valor de la resistividad

$\rho=\frac{2 \pi(3 m)(8.4 \Omega)}{\ln \left(\frac{8 * 3 m}{0.013 m}\right)-1}=21.85 \Omega \cdot m$

\section{Mediciones iniciales de Resistencia}

Se realizaron las mediciones de la conexión a tierra para cada sistema (ver tabla 2). 


\begin{tabular}{|l|l|l|}
\hline Edificio & Subestación $(\mathrm{kVA})$ & Resistencia $(\boldsymbol{\Omega})$ \\
\hline A & 150 & 9.5 \\
\hline B & 150 & 10.6 \\
\hline C y D & 225 & 8.4 \\
\hline E y F & 150 y 225 & 12.3 \\
\hline
\end{tabular}

Tabla 2 Mediciones en subestaciones Fuente: Elaboración Propia

De acuerdo con la tabla anterior, existen valores de resistencia de cada sistema de puesta a tierra que cumplen (edificios A, C y D) y otros que no cumplen (edificios B, E y F) con la NOM001-SEDE-2012 ni con las Especificaciones CFE 01J00_01, que es un valor máximo de $10 \Omega$ en época de estiaje. Ningún sistema cumple la exigencia de la norma IEEE-Std-80-2002, la cual se muestra en las siguientes tablas.

\begin{tabular}{|l|l|}
\hline \multicolumn{1}{|c|}{ Tipo de instalación eléctrica } & $\begin{array}{c}\text { Resistencia de } \\
\text { puesta a tierra } \\
{[\Omega]}\end{array}$ \\
\hline $\begin{array}{l}\text { Subestaciones de gran tamaño }(20,000 \\
\left.\mathrm{m}^{2}\right) \text { y de Transmisión }\end{array}$ & 1 o menos \\
\hline $\begin{array}{l}\text { Subestaciones de plantas industriales, } \\
\text { edificios e instalaciones comerciales } \\
\text { grandes y subestaciones pequeñas }\end{array}$ & Rango de 1 a 5 \\
\hline $\begin{array}{l}\text { Electrodos individuales } \\
\text { (residenciales) }\end{array}$ & 25 \\
\hline Torres de transmisión individuales & 10 \\
\hline
\end{tabular}

Tabla 3 Valores de resistencia de puesta a tierra. Fuente: IEEE-Std-80-2000

\begin{tabular}{|r|r|}
\hline \multicolumn{1}{|c}{$\begin{array}{c}\text { Capacidad de la } \\
\text { Subestación [MVA] }\end{array}$} & $\begin{array}{c}\text { Resistencia de la Red de } \\
\text { tierra }[\Omega]\end{array}$ \\
\hline$\leq 0.05$ & 12 \\
\hline $0.05-0.1$ & 6 \\
\hline $0.1-0.5$ & 2 \\
\hline $0.5-1$ & 1.5 \\
\hline $1-50$ & 1 \\
\hline $50-100$ & 0.5 \\
\hline$>100$ & 0.2 \\
\hline
\end{tabular}

Tabla 4 Valores recomendados de la resistencia de puesta a tierra para subestaciones

Fuente: IEEE-Std-80-2000

Derivado de lo anterior, cuando se presentan descargas atmosféricas o variaciones de voltaje, se dañan equipos eléctricos, principalmente equipo de cómputo y telecomunicaciones. Debido a esta problemática, se diseña un nuevo sistema de puesta a tierra tipo malla.

Se considera construir una red de tierras equipotencial para las cinco subestaciones de la Facultad, pero debido al bajo presupuesto, sólo se consideraron tres subestaciones, las más críticas.
La corriente de corto circuito $\left(I_{c c}\right)$ será calculada a partir de la potencia de corto circuito que proporciona la compañía suministradora la cual es de 800 MVA.

Debido a que la condición más crítica de corriente de cortocircuito en la subestación es producida por una falla de fase a tierra en la barra de 220/127V, la $I_{c c}$ será calculada con la siguiente ecuación

$$
I_{c c}=\frac{M V A_{c c}}{\sqrt{3}(k V)}[k A]
$$

sustituyendo los datos, tenemos

$I_{c c}=\frac{800 M V A}{\sqrt{3}(13.8 k V)}=33.469 k A$

Considerando un factor de crecimiento a futuro del sistema, es decir $f_{c}=1.3$, la corriente de falla simétrica eficaz (rms) para el diseño de la red es

$I_{f}=f_{c} * I_{c c}$

Para nuestro sistema, $I_{f}$ es

$I_{f}=1.3 * 33.469=43.51 k A$

\section{Cálculo de la sección transversal del conductor}

La sección transversal del conductor para un sistema de tierra requerida, en función de la elevación de temperatura de corto tiempo, la magnitud y el tiempo de duración de la falla y cuando se conocen las constantes el material del conductor, se puede determinar con la siguiente ecuación, de acuerdo con Sverak (IEEE Std 8371989, Apéndice B)

$I_{f}=A \sqrt{\left[\frac{T C A P * 10^{-4}}{t_{c} \sigma_{r} \rho_{r}}\right] \ln \left[\frac{K_{0}+T_{m}}{K_{0}+T_{a}}\right]}$

donde: $I_{f}=$ Corriente de falla rms en $\mathrm{Ka}$

$A=$ Sección transversal del conductor en $\mathrm{mm}^{2}$

$\mathrm{Tm}=$ Temperatura máxima permisible en ${ }^{\circ} \mathrm{C}$

$T a=$ Temperatura ambiente en ${ }^{\circ} \mathrm{C}$

$\operatorname{Tr}=$ Temperatura de referencia para las constantes del material en ${ }^{\circ} \mathrm{C}$

$\sigma_{0}=$ Coeficiente térmico de la resistividad a $0^{\circ} \mathrm{C}$ en $1 /{ }^{\circ} \mathrm{C}$

$\sigma_{r}=$ Coeficiente térmico de la resistividad a la temperatura de referencia $\operatorname{Tr}$ en $1 /{ }^{\circ} \mathrm{C}$

LEZAMA-ZÁRRAGA, Francisco Román, OVANDO-SIERRA, Juan Carlos, CASTILLO-TÉLLEZ, Margarita y ANDRADE-DURÁN, Juan Edgar. Estudio y diseño del Sistema de Tierra Integral de la Facultad de Ingeniería de la Universidad Autónoma de Campeche. Revista de Ingeniería Eléctrica. 2019 
$\rho_{r}=$ Resistividad del conductor de puesta a tierra a la temperatura de referencia Ta en $\mu \Omega$-cm $K_{0}=1 / \sigma_{r}$ en ${ }^{\circ} \mathrm{C}$ $t_{c}=$ Duración de la corriente en segundos $T C A P=$ Capacidad térmica por unidad de volumen, en $\mathrm{J} / \mathrm{cm}^{3} /{ }^{\circ} \mathrm{C}$.

Para conductores de cobre a cierta temperatura de referencia y con la conductividad de 97\%, se tiene los siguientes valores (IEEE,2000)

$\sigma_{r}=0.003811 /{ }^{\circ} \mathrm{C}^{\operatorname{conT}} \mathrm{T}_{r}=20^{\circ} \mathrm{C}$

$K_{0}=242^{\circ} \mathrm{C}$

$T_{m}=1084^{\circ} \mathrm{C}$

$\rho_{r}=1.78 \mu \Omega-\operatorname{cmconT}_{r}=20^{\circ} \mathrm{C}$

TCAP $=3.42^{\mathrm{J}} /\left(\mathrm{cm}^{3 \circ} \mathrm{C}\right)$

$T_{a}=29^{\circ} \mathrm{C}$

De la ecuación (4), despejando el área A de la sección transversal del conductor, tenemos $A=\frac{I_{f}}{\sqrt{\left[\frac{T C A P * 10^{-4}}{t_{c} \sigma_{r} \rho_{r}}\right] \ln \left[\frac{K_{0}+T_{m}}{K_{O}+T_{a}}\right]}}$

sustituyendo los valores del conductor,

$A=\frac{43.51}{\sqrt{\left[\frac{3.42 \times 10^{-4}}{0.5 * 0.00381 * 1.78}\right] \ln \left[\frac{242+1084}{242+29}\right]}}=\frac{43.51}{\sqrt{0.1078 * 1.587}}=$ $105.17 \mathrm{~mm}^{2}$

Por lo tanto, para esta área de la sección del conductor, tenemos un calibre

$A_{T}=105.17 \mathrm{~mm}^{2} \rightarrow \mathrm{Cal}^{4} /{ }_{0} A W G$

Con la sección transversal mínima obtenida se puede seleccionar el conductor de sección transversal inmediata superior existente de acuerdo con la norma NOM-001-SEDE-2012 lo que resulta ser de $107.2 \mathrm{~mm}^{2}$ (4/0 AWG) de cobre, siendo este calibre idóneo por su resistencia mecánica, mejor conductividad tanto eléctrica como térmica y sobre todo por ser resistente a la corrosión debido a que es catódico respecto a otros materiales que pudieran estar enterrados cerca de este conductor.

\section{Límite de Tensiones de paso y de contacto}

Siguiendo la metodología de IEEE-Std-80-2000, se procede a calcular los potenciales de paso y de contacto, considerando una duración de falla de $0.5 \mathrm{~s}$ y una resistividad promedia del terreno de $\rho=21.85 \Omega-\mathrm{m}$.
Para el diseño se considera que no se tiene una capa superficial por lo que: $\rho_{s}=\rho \mathrm{y}$ $C_{s}=1$.

La tensión de paso para $50 \mathrm{~kg}$ está dada por:

$E_{\text {paso }}=\left(1000 \Omega+\left(6 C_{s} * \rho_{s}\right) \frac{0.116}{\sqrt{t_{s}}}\right.$

Sustituyendo los valores, tenemos

$$
\begin{gathered}
E_{\text {paso }}=\left(1000 \Omega+\left(6 C_{S} * \rho_{S}\right) \frac{0.116}{\sqrt{t_{s}}}\right. \\
E_{\text {paso }}=\left(1000+\left((6 * 1) *(21.85) \frac{0.116}{\sqrt{0.5}}\right.\right. \\
=(1000+131.1) * 0.1640
\end{gathered}
$$

$E_{\text {paso }}=185.5 \mathrm{~V}$

Asimismo, la tensión de contacto para 50 $\mathrm{kg}$ esta dada por

$$
E_{\text {contacto }}=\left(1000 \Omega+\left(1.5 C_{s} * \rho_{s}\right) \frac{0.116}{\sqrt{t_{s}}}\right.
$$

Sustituyendo los datos, tenemos

$$
\begin{aligned}
E_{\text {contacto }}= & \left(1000+\left((1.5 * 1) *(21.85) \frac{0.116}{\sqrt{0.5}}\right.\right. \\
= & (1000+32.78) * 0.1640 \\
E_{\text {contacto }}= & 169.37 \mathrm{~V}
\end{aligned}
$$

Estos valores cumplen con los limites normativos de acuerdo con la tabla 4 (IEEE, 2000).

\begin{tabular}{|r|r|r|}
\hline \multicolumn{1}{l|}{$\begin{array}{l}\text { Tiempo } \\
\text { (s) }\end{array}$} & $\begin{array}{l}\text { Tensión máxima } \\
\text { de paso }(\mathrm{V})\end{array}$ & $\begin{array}{l}\text { Tensión máxima de } \\
\text { contacto }(\mathrm{V})\end{array}$ \\
\hline$>5$ & 500 & 50 \\
\hline $3-5$ & 640 & 64 \\
\hline 2 & 690 & 69 \\
\hline 1 & 785 & 78.5 \\
\hline 0.9 & 800 & 80 \\
\hline 0.7 & 1020 & 102 \\
\hline 0.5 & 1440 & 194 \\
\hline 0.2 & 3600 & 360 \\
\hline$\leq 0.1$ & 7200 & 720 \\
\hline
\end{tabular}

Tabla 5 Valores de tensión máximos admisibles para personas de $50 \mathrm{~kg}$ y $70 \mathrm{~kg}$

Fuente: IEEE-Std-80-2000

\section{Longitud total del conductor de la malla}

La cantidad de conductor de la red de tierras es su longitud total. Para determinarla se debe procurar que las mallas formen lo más aproximado posible un cuadrilátero. 
Por lo que la relación entre el número de mallas sobre el eje "X" y el número de mallas sobre el eje "Y" debe corresponder aproximadamente a la relación entre el largo y el ancho de la red.

$\frac{\text { eje } \mathrm{x}}{\text { eje } \mathrm{y}}=\frac{\text { largo de la red }}{\text { ancho de la red }}=\frac{4}{3}=1.33$

Si en forma tentativa se consideran 5 hilos sobre el eje " $x$ ", el número de hilos sobre el eje "y" será:

hiloseje"y" $=\frac{5}{1.33}=3.76 . \approx 4$

Por lo que en forma preliminar la red estará constituida por 5 conductores transversales (paralelos al eje "y"), y de 4 conductores longitudinales (paralelos al eje " $\mathrm{x}$ "), con una longitud total $\left(\mathrm{L}_{\mathrm{T}}\right)$ :

$L_{T}=\left(a * L_{x}\right)+\left(b * L_{y}\right)+L_{\text {punta }}$

Sustituyendo valores

$L_{T}=(4 * 3 m)+(5 * 4 m)=32 m$.

Con una separación preliminar entre los conductores paralelos igual a

$D=\frac{2 \mathrm{~L}_{x} * l_{y}}{L_{C}-L_{X}-L_{Y}}$

Y así, obtenemos

$D=\frac{(2 * 4 * 3)}{32-4-3}=0.96 \mathrm{~m} . \approx 1 \mathrm{~m}$.

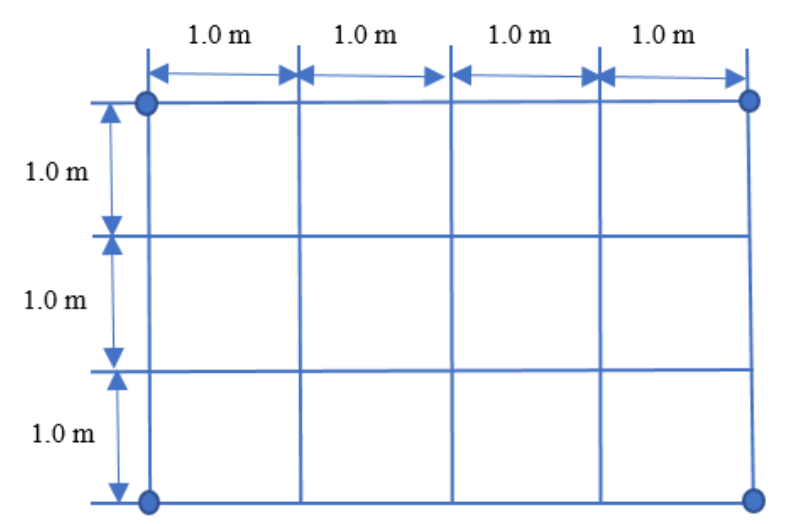

Figura 5 Dimensiones de la malla de tierra Fuente: Elaboración Propia

Características de la malla:

A: Longitud de la malla $(\mathrm{A}=4 \mathrm{~m})$

B: Ancho de la malla $(B=3 \mathrm{~m})$

L: Longitud del conductor $(\mathrm{L}=32 \mathrm{~m})$ n: Número de conductores en paralelos de longitud A $(n=4)$

$\mathrm{m}$ : Número de conductores en paralelo de longitud $B(m=5)$

D: Espaciamiento entre conductores $(\mathrm{D}=1 \mathrm{~m})$

$\mathrm{h}$ : Profundidad de enterramiento $(\mathrm{h}=0.60 \mathrm{~m})$

$\mathrm{d}$ : Diámetro del conductor $(\mathrm{d}=11.68 \mathrm{~mm}=$ $0.01168 \mathrm{~m})$.

\section{Resistencia de Puesta a Tierra}

Para calcular la resistencia de puesta a tierra utilizaremos el método de Laurent y Niemann, el cual tiene la siguiente ecuación para su cálculo

$R=\frac{\rho}{4 r}+\frac{\rho}{L}$

donde:

$r=\sqrt{\frac{A}{\pi}}$ y es conocido como radio equivalente.

$\mathrm{L}=$ longitud del conductor

$\mathrm{A}=(3.0 \mathrm{~m} . \mathrm{x} 4.0 \mathrm{~m})=.12.0 \mathrm{~m}^{2}$

Tenemos que

$r=\sqrt{\frac{12}{\pi}}=1.954 \mathrm{~m}$.

Por lo tanto:

$R=\frac{21.85}{4(1.954)}+\frac{21.85}{32}=2.79+0.68=3.47 \Omega$

Este valor cumple con la normativa de la tabla 3 para las subestaciones de la Facultad: 150 kVA y 225 kVA.

\section{Número mínimo de varillas}

El número mínimo de varillas que requerido es:

$N v=0.60 \sqrt{A}$

Entonces tenemos que

$N v=0.60 \sqrt{12}=2$

El número de varillas que se instalarán en total son 4 por lo que se cumple con el valor calculado.

\section{Resultados}

Se instalaron tres mallas de puesta a tierra, para los edificios más críticos, tal como se muestra en la figura 6. 


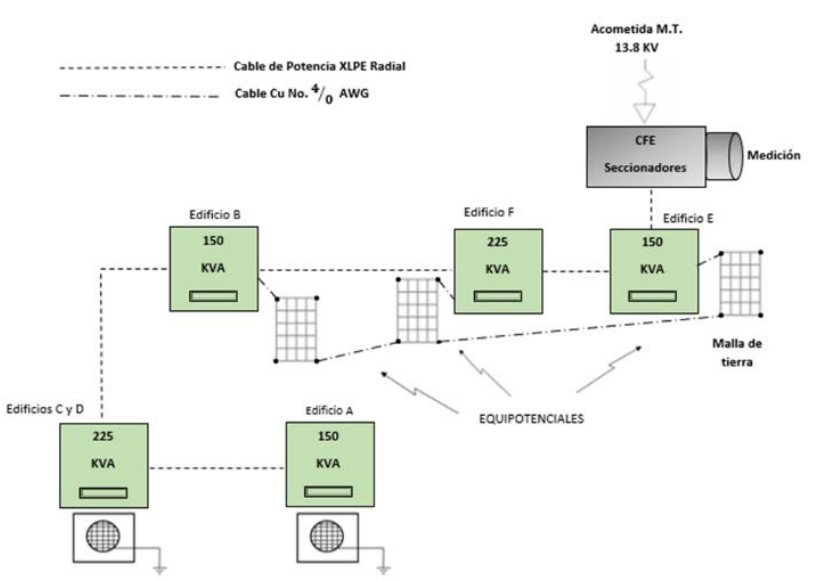

Figura 6 Sistema de puesta a tierra actual. Fuente: Elaboración propia

Se realizaron las mediciones al sistema interconectado y equipotencial formado por los edificios B, E y F para comparar con las mediciones iniciales mostradas en la tabla 1 .

\begin{tabular}{|l|r|r|}
\hline Edificio & \multicolumn{1}{|r|}{ Subestación $(\mathrm{kVA})$} & Resistencia $(\boldsymbol{\Omega})$ \\
\hline A & 150 & 9.7 \\
\hline B & 150 & 3.6 \\
\hline C y D & 225 & 8.8 \\
\hline E & 150 & 3.7 \\
\hline F & 225 & 3.9 \\
\hline
\end{tabular}

Tabla 6 Nuevas mediciones en subestaciones Fuente: Elaboración Propia

Las mediciones muestran que el sistema interconectado formado por las mallas de los edificios B, E y F son equipotenciales. En los edificios $\mathrm{A}, \mathrm{C}$ y $\mathrm{D}$ no se realizaron modificaciones por lo que se mantuvieron las mediciones iniciales ya que son sistemas de puesta a tierra con electrodo e independientes.

\section{Conclusiones y trabajos futuros}

En este artículo se realizó el diseño e implementación de un sistema de tierras integral y equipotencial en la Facultad de Ingeniería para proporcionar condiciones de seguridad a las personas y equipos que operan en la instalación eléctrica en baja tensión, drenando rápidamente la falla, logrando que el valor del voltaje con respecto a la tierra que se alcance en la parte fallada sea igual o inferior al valor de seguridad.

Mediante las mediciones, primero, de resistencia del suelo, se obtuvo el nivel de resistividad que fue el punto de partida de nuestro diseño; segundo, las mediciones de nivel de resistencia de puesta a tierra de las cinco subestaciones nos mostraron que no cumplían con la normativa vigente.
Por lo que era común el daño permanente a equipos de cómputo y telecomunicaciones. Se implementó el sistema de tierra integral, sólo de las tres subestaciones más críticas, con el mayor valor de resistencia de puesta a tierra y se logró alcanzar los valores normativos. Se demuestra que al implementar estas medidas las condiciones de seguridad son confiables para los seres humanos y los equipos y que se le está dando el interés debido al sistema de puesta a tierra.

Como acciones futuras, se pretende en un corto plazo integrar al sistema de puesta a tierra a los edificios A, C y D, para tener seguridad y confiabilidad en todo el campus de la facultad de Ingeniería y elaborar un programa de mantenimiento preventivo.

Se concluye que la metodología empleada es una guía de acciones que se deben realizar de manera ordenada y con un orden cronológico en Instituciones de Educación Superior con el fin de optimizar el sistema de puesta a tierra.

\section{Referencias}

Asociación de Normalización y Certificación A. C. (2005). Norma NXM-J-549-ANCE-2005, Sistema de protección contra tormentas eléctricas. México.

Baca, L. F. G., Flores, S. C., Medina-González, I., Olvera, A. A., Hermanson, P. M., Solís, C. A. G., \& Glantz, R. S. (2019). Conservación de bienes culturales: acciones y reflexiones. Instituto Nacional de Antropología e Historia

Cabrera Dávila, G. D. L. C. (2019). Evaluación de la macrofauna edáfica como bioindicador del impacto del uso y calidad del suelo en el occidente de Cuba. Tesis Doctoral. Universidad de Alicante. España.

Checkland, P. \& Scholes J. (1994). Metodología de los sistemas suaves en acción. México: Megabyte, Noriega Editores. 352p.

Comisión Federal de Electricidad. (2015). Especificaciones CFE 01J00_01. México. 
Dawalibi, F. \& Mukhedkar, D. (1979) Resistance measurement of large grounding systems, IEEE Transactions on Power Apparatus and Systems, November/December, Vol. PAS98, No. 6, pp.2348-2354.

Díaz, P. (2001). Soluciones prácticas para la puesta a tierra de sistemas eléctricos. México: Editorial Mc Graw Hill.

Estevez L. (2012) NOM 001 Sede 2012 Instalaciones Eléctricas (Utilización). México: Limusa.

García Márquez, R. (2001). La puesta a tierra de instalaciones eléctricas. México: Alfaomega Marcombo.

Guemes, J. A. \& Hernando, F. E. (2004). Method for calculating grounding resistance of grounding grids using FEM, IEEE Transactions on Power Delivery, April, Vol. 19, No. 2, pp.595-600.

Herrera, J. A. \& Castro, O. (2003). Cálculo de la malla de puesta a tierra de una subestación. Scientia et Technica, Año IX, No. 22, Colombia: Redalyc.

León, M., \& Roger, W. (2019). Índice de diversidad de flora silvestre y concentración de metales pesados del suelo asociados a los Géiseres de la Micro Cuenca de Calientes, Provincia de Candarave-Tacna. Tesis Doctoral. Universidad Nacional Jorge Basadre Grohmann. Perú.

Markiewicz, H. \& Klajn, A. (2003). Puestas a tierra $y$ EMC. Wroclaw University of Technology. Reino Unido; Leonardo Power Quality Initiative.

Melgar Chipana, A., Yactayo, Y., \& Daniel, J. (2019). Análisis descriptivo del cumplimiento del plan estratégico del sector pesquero peruano en el periodo 2012 al 2016. Tesis de Licenciatura. Universidad Autónoma de Perú. Perú.

Mollo, A., \& Benjamin, M. (2019). Estudio Geofísico de Resistividad Eléctrica aplicando la configuración Dipolo-Dipolo para localización de cavidades en el municipio de solidaridad, playa del Carmen, estado de Quintana, ROO, Mexico. "Proyecto Predio 3.04 HAS".
The Institute of Electrical and Electronics Engineers, Inc. (2000). Norma ANSI/IEEE-Std80-2000, IEEE Guide for Safety in AC Substation Grounding. USA.

The Institute of Electrical and Electronics Engineers, Inc. (2012). Norma ANSI/IEEE-Std81-2012, IEEE Guide for Measurement of Impedance and Safety Characteristics of Large, Extended or Interconnected Grounding Systems. USA.

The Institute of Electrical and Electronics Engineers, Inc. (1989). Norma ANSI/IEEE Std 837-1989, IEEE Standard for Qualifying Permanent Connections Used in Substation Grounding. USA.

Velasco Solís, J. (2007). Entendiendo el sistema de tierras basado en la NOM. México: Ingeniería Mecánica Industrial. 